\title{
Finite Analytic Model for Flow and Transport IN UNSATURATED ZONE
}

\author{
By Whey-Fone Tsai, ${ }^{1}$ Tim-Hau Lee, ${ }^{2}$ Ching-Jen Chen, ${ }^{3}$ Shin-Jye Liang, ${ }^{4}$ and Chia-Chen Kuo ${ }^{5}$
}

\begin{abstract}
The finite analytic method is employed to solve the vertical, two-dimensional subsurface flow and transport equations in an unsaturated zone. The finite analytic method treats the nonlinear coefficient terms of the governing equations as constants in the element so that linearized partial differential equations can be obtained and solved in each element. The accuracy and limitations of the numerical method are systematically explored. The flow and transport simulations are examined using a one-dimensional laboratory infiltration test and an analytical solution of a two-dimensional subsurface transport problem, respectively. In the advection-dominant, vertical, one-dimensional infiltration problem, nine spatial weighting schemes are proposed to evaluate the averaged unsaturated hydraulic conductivity in a discretized element. Among them, the geometric mean weighting scheme provides the most accurate results as compared with the infiltration data. In verification of the two-dimensional solute transport problem, the nine-node elements are placed in the interior domain, and different layers of five-node elements are placed at the boundaries to investigate if the numerical experiment setup was proper and the algorithm was accurate. The developed numerical model is then applied to an irregular-domain landfill leaching problem to reveal the features of subsurface transport in unsaturated zone. Numerical aspects to be further explored are suggested.
\end{abstract}

\section{INTRODUCTION}

In the subsurface, pollutant moves with water flow by several processes, including advection, diffusion, retardation, and decay. To investigate the consequence of the hazard posted by solute, it is important to look into the problems of water and solute migration in the unsaturated zone. In mathematical terms, solute transport in the subsurface is described by the advection-diffusion differential equation. The equations for flow and associated solute transport in the unsaturated zone along the vertical direction are highly nonlinear. Particularly, the nonlinear coefficient terms are functions of the dependent variables and are very sensitive to the dependent variables. During infiltration, the large gradients of pore pressure and hydraulic conductivity make the numerical simulation in an unsaturated zone even more difficult. The accuracy of numerical solutions may be deteriorated if an improper numerical algorithm is used.

Recently, a nontraditional, analytical-solution based numerical technique named the finite analytic method (Chen and Chen 1984a,b; Chen 1989) has attracted various researchers in the environmental engineering community (Hwang et al. 1985; Li et al. 1989; Tsai et al. 1993; Pi and Hjelmfelt 1994). The method combines numerical flexibility with the advantages of analytical solutions such that the truncation errors occurring in other numerical methods can be minimized and the numerical solutions are quite stable (Zeng and Li 1987). Hwang et al. (1985) first used the finite analytic method of Chen (1989) for solving the horizontal, two-dimensional solute transport equation. In the finite analytic method, the coefficients of the governing equations are frozen in each subdomain so that linear partial differential

${ }^{1}$ Res. Sci., CFD \& Envir. Engrg. Group, Nat. Ctr. for High-Performance Computing, No. 7 R\&D 6th Rd., Hsinchu Science-Based Industrial Park, Shinchu, Taiwan.

${ }^{2}$ Assoc. Prof., Dept. of Civ. Engrg., Nat. Taiwan Univ., Taipei, Taiwan.

${ }^{3}$ Prof., Dept. of Mech. Engrg., Florida A\&M Univ./Florida State Univ., Tallahassee, FL 32316.

${ }^{4}$ Res. Sci., CFD \& Envir. Engrg. Group, Nat. Ctr. for High-Performance Computing, No. 7 R\&D Rd., Hsinchu Science-Based Industrial Park, Hsinchu, Taiwan.

${ }^{5}$ Res. Sci., CFD \& Envir. Engrg. Group, Nat. Ctr. for High-Performance Computing, No. 7 R\&D Rd., Hsinchu Science-Based Industrial Park, Hsinchu, Taiwan.

Note. Associate Editor: Alexander Cheng. Discussion open until October 1, 2000. To extend the closing date one month, a written request must be filed with the ASCE Manager of Journals. The manuscript for this paper was submitted for review and possible publication on March 8, 1999. This paper is part of the Journal of Engineering Mechanics, Vol. 126, No. 5, May, 2000. (C)ASCE, ISSN 0733-9399/00/0005-0470$0479 / \$ 8.00+\$ .50$ per page. Paper No. 20423. equations can be solved in each element subject to appropriate boundary conditions at the element's boundaries. Li et al. (1989) employed the finite analytic method to the two-dimensional irrotational gravity flows over a spillway with a free surface. The coupled solutions of free surface level and flow rate were solved and in good agreement with experimental measurement. Pi and Hjelmfelt (1994) used a hybrid finite analytic method to solve the lateral subsurface storm flow described by the extended DuPuit-Forchheimer equation. They concluded that a four-point numerical formula could provide results that are stable and accurate without using small time steps. In addition to the reports indicated above, the treatment of irregular boundaries using the finite analytic method associated with mixed nine-node and five-node elements and solving the system of algebraic equations on a Cartesian grid system was developed by Tsai et al. (1993) for unsaturated flow problems. One of the common aspects among the reviewed literature was results in a matrix of a positive nature. Leonard and Mokhtari (1990) indicated that the hybrid finite analytic method is equivalent to the first-order upwinding scheme for advection with physical diffusion omitted when the Peclet number is over two, and the advantage of the finite analytic method is its property to increase the mesh Peclet number of stability.

The present study, therefore, is aimed at solving the subsurface flow and solute transport equations in an unsaturated zone by using the finite analytic method. In an unsaturated zone, the sharp wetting front may cause large velocity gradients along the vertical direction. Hence, proper numerical manipulation is required to get through the numerical difficulties associated with the sharp front capturing. Since both flow and transport in the subsurface are advection-diffusion problems, the same numerical formulation can be used. Also, for the purpose of field applications, we should take the irregularity of computational domain into account in the numerical formulation. The viewpoints noted above, together with verifications, applications, and features of the numerical model, are explored in this study.

\section{GOVERNING EQUATIONS}

\section{Vertical Two-Dimensional Subsurface Flow in Unsaturated Zone}

The Richards equation (Bear 1979) describing transient, two-dimensional subsurface flow in an unsaturated zone, or unsaturated flow, is 


$$
C_{s} \frac{\partial H}{\partial t}-\frac{\partial}{\partial x}\left(K \frac{\partial H}{\partial x}\right)-\frac{\partial}{\partial z}\left(K \frac{\partial H}{\partial z}\right)-\frac{\partial K}{\partial z}=0
$$

Eq. (1) assumes that air pressure is negligible and that there is no sink or source in the flow domain. The hydraulic conductivity in the unsaturated zone, $K$, is isotropic, and the porosity and water density in the porous media remain constant. $H=$ pressure head; $C_{s}=$ specific moisture capacity, which is a nonlinear function of $H$ and is defined as $\partial \theta(H) / \partial H$; and $\theta$ $=$ volumetric water content. $K$ and $\theta$ are both the nonlinear functions of $H$. The two functions can be determined by laboratory experiments. $x$ and $z=$ horizontal and vertical (positive upward) space coordinates, respectively; and $t=$ time.

\section{Vertical Two-Dimensional Subsurface Transport in Unsaturated Zone}

The governing equation for the vertical two-dimensional subsurface solute transport in an unsaturated zone (Bear 1979) can be written as

$$
\begin{aligned}
& \frac{\partial(R \theta C)}{\partial t}+\frac{\partial\left(V_{x} \theta C\right)}{\partial x}+\frac{\partial\left(V_{z} \theta C\right)}{\partial z}=\frac{\partial}{\partial x}\left[D_{x x} \frac{\partial(\theta C)}{\partial x}\right] \\
& +\frac{\partial}{\partial x}\left[D_{x z} \frac{\partial(\theta C)}{\partial z}\right]+\frac{\partial}{\partial z}\left[D_{z z} \frac{\partial(\theta C)}{\partial z}\right]+\frac{\partial}{\partial z}\left[D_{z x} \frac{\partial(\theta C)}{\partial x}\right] \\
& -\lambda R \theta C+s
\end{aligned}
$$

in which $C=$ solute concentration; $R=$ retardation factor due to sorption of soil; $V_{x}$ and $V_{z}=$ porous velocity components of unsaturated flow in the $x$ and $z$ directions, respectively; $D_{x x}, D_{z z}, D_{x z}$, and $D_{z x}=$ coefficients of mechanical dispersion provided that the contribution of molecular diffusion to dispersion is neglected; $\lambda=$ first-order decay coefficient; and $s=$ solute source-sink term. $V_{x}, V_{z}$, and $\theta$ in (2) can be obtained from the solution of the unsaturated flow equation, (1). Mathematically, the dependent variable $C$ of (2) can incorporate $\theta$ and form a new dependent variable $C^{*}=C \theta$. The parameters $R, D_{x x}, D_{z z}, D_{x z}, D_{z x}$, and $\lambda$ are described in the following. The retardation factor, $R$, is defined as

$$
R=1+\frac{K_{d} \rho_{b}}{\theta}
$$

in which $K_{d}=$ equilibrium distribution coefficient, which is the mass of chemical adsorbed to the solid phase per unit mass of solid phase divided by the concentration of the chemical in the water; and $\rho_{b}=$ bulk density of soil. The coefficients of mechanical dispersion (Anderson 1979) can be expressed by

$$
\begin{aligned}
& D_{x x}=\alpha_{1} \frac{V_{x}^{2}}{\sqrt{V_{x}^{2}+V_{z}^{2}}}+\alpha_{t} \frac{V_{z}^{2}}{\sqrt{V_{x}^{2}+V_{z}^{2}}} \\
& D_{z z}=\alpha_{t} \frac{V_{x}^{2}}{\sqrt{V_{x}^{2}+V_{z}^{2}}}+\alpha_{1} \frac{V_{z}^{2}}{\sqrt{V_{x}^{2}+V_{z}^{2}}}
\end{aligned}
$$

and

$$
D_{x z}=\left(\alpha_{1}-\alpha_{t}\right) \frac{V_{x} V_{z}}{\sqrt{V_{x}^{2}+V_{z}^{2}}}=D_{z x}
$$

in which $\alpha_{1}$ and $\alpha_{t}$ denote longitudinal and transversal dispersivities, respectively. The first-order decay coefficient $\lambda$ can be related to the half-life time, $T_{1 / 2}$, by

$$
\lambda=\frac{\ln 2}{T_{1 / 2}}
$$

\section{FINITE ANALYTIC NUMERICAL SOLUTIONS}

In this section, the finite analytic numerical solutions incorporating a valuable time-weighting factor are derived for both unsaturated flow and solute transport equations for both ninenode and five-node local elements. The nine-node elements are placed in the regular boundaries and interior domain, while the five-node elements are placed at the irregular boundaries (Tsai et al. 1993). For the dependent variable $\Phi(\Phi=H$ or $\Phi$ $\left.=C^{*}=C \theta\right)$ the time-weighting factor associated with the unsteady term, $(\partial \Phi / \partial t)$, within one time step can be expressed by

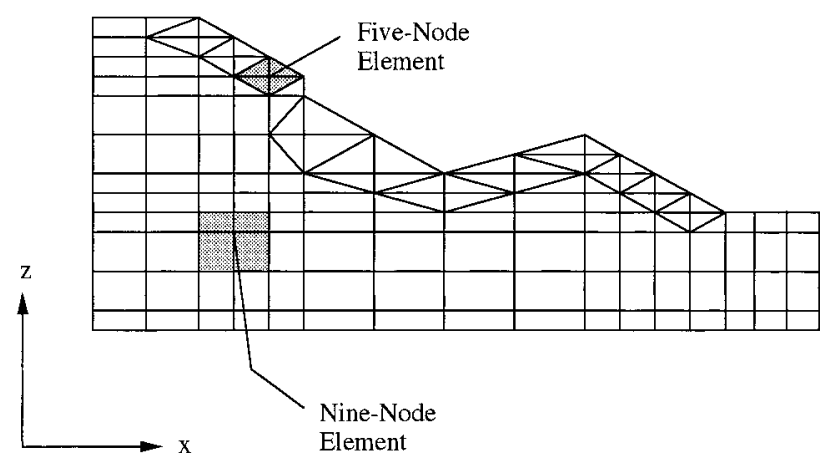

FIG. 1. Nine-Node and Five-Node Local Elements in Domain with Irregular Boundaries
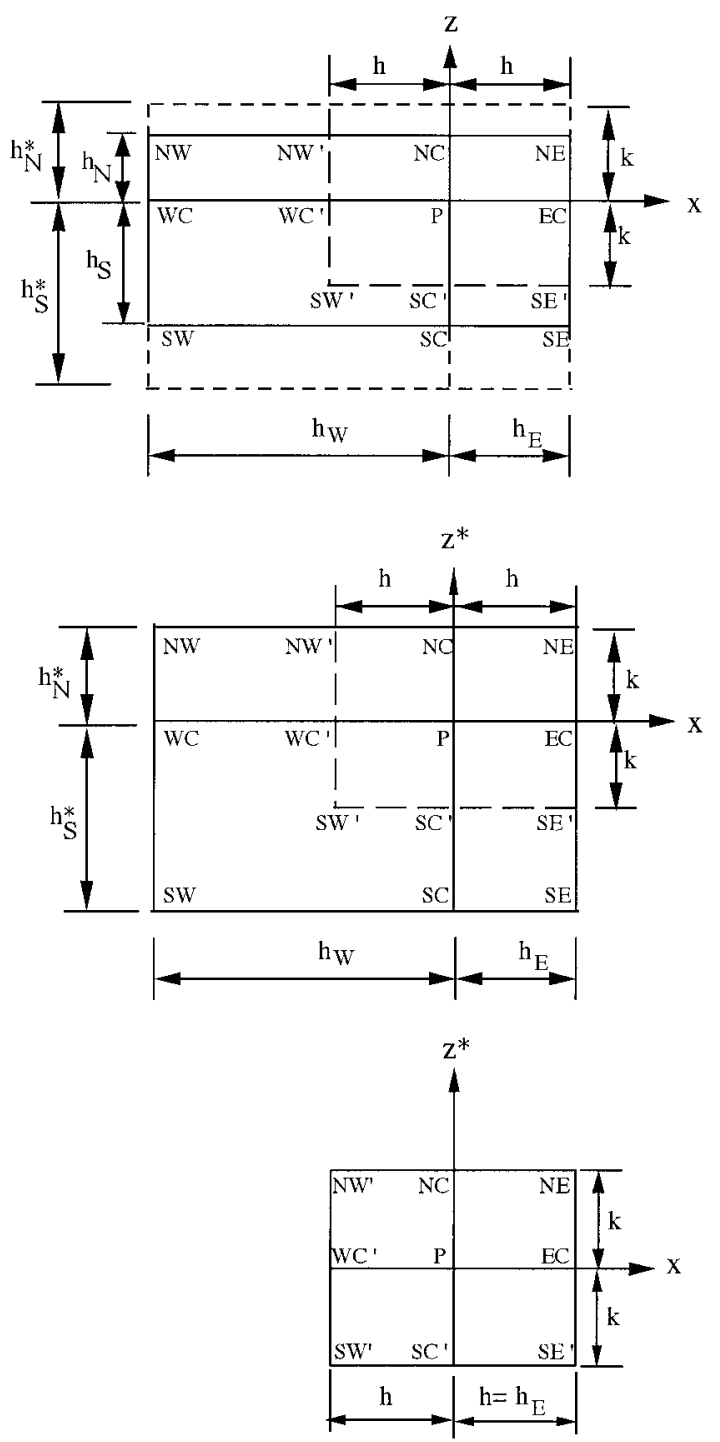

FIG. 2. Numerical Solution Procedures for Nine-Node Element: (a) in Original $x-z$ Coordinate System; (b) Transformation from $z$ Coordinate System to $z^{*}$ Coordinate System; (c) UniformGrid Element Used to Provide Analytical Solution 


$$
\frac{\Phi^{n}-\Phi^{n-1}}{\Delta t}=\omega\left(\frac{\partial \Phi}{\partial t}\right)^{n-1}+(1-\omega)\left(\frac{\partial \Phi}{\partial t}\right)^{n}
$$

in which $\Delta t=$ time interval used for computation; $n=$ time level; and $\omega=$ time-weighting factor. The optimal value of $\omega$, was proposed by Tsai et al. (1993) based on investigation of the analytical solutions of a one-dimensional advection-diffusion equation, can be written as

$$
\omega=0.5\left[\left(\frac{\partial \Phi}{\partial t}\right)^{n-1} /\left(\frac{\Phi^{n}-\Phi^{n-1}}{\Delta t}\right)\right]^{-0.28}
$$

The magnitude of $\omega$ is in a range of $0-1$.

In the finite analytic method, the computational domain is subdivided into small elements, as sketched in Fig. 1. The local element is either a nine-node element (Fig. 2) placed in the interior domain and regular boundaries or a five-node element (Fig. 3) placed at the irregular boundaries. The unsteady term in the governing equations, $(\partial \Phi / \partial t)^{n}$, can be substituted by the other terms of (8) such that the time-weighting factor $\omega$ can be linked to the formulation. Consequently, either the nine-node element or the five-node element [(1) and (2)] can be locally linearized and expressed by

$$
\frac{\partial^{2} \Phi^{n}}{\partial x^{2}}+G \frac{\partial^{2} \Phi^{n}}{\partial z^{2}}=2 A \frac{\partial \Phi^{n}}{\partial x}+2 B G \frac{\partial \Phi^{n}}{\partial z}+g
$$

with

$$
g=\operatorname{Re} \frac{\Phi_{p}^{n}-\Phi_{p}^{n-1}}{\left(1-\omega_{p}\right) \Delta t}+f_{p}
$$

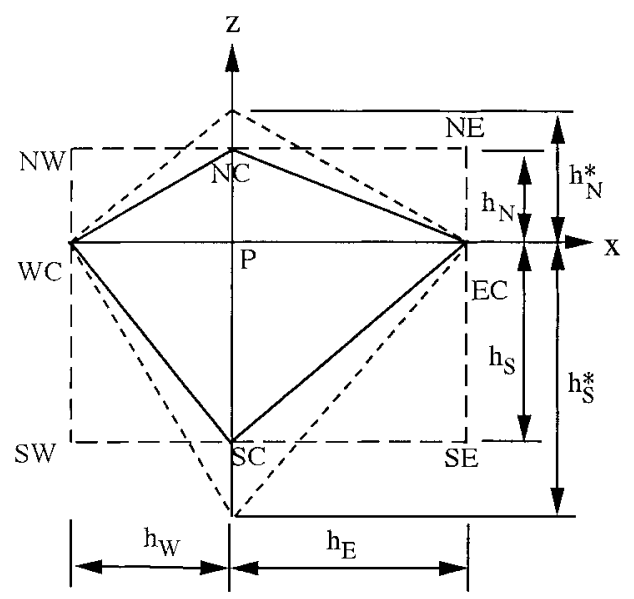

(a)

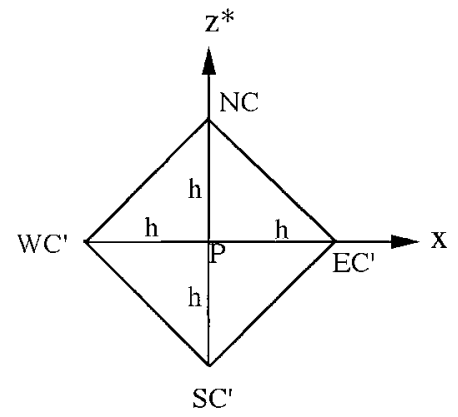

(c)
The expressions of $G, A, B, g, R e$, and $f_{p}$ in (10) and (11) for both unsaturated flow and solute transport equations are tabulated in Table 1 . In the finite analytic method, terms $G, A$, and $B$ are taken to be constants in the element for each time level of calculation. The term $g$ is also taken to be a constant in the numerical formulation, but it is decomposed into two terms respectively associated with $\Phi_{p}^{n-1}$ and $\Phi_{p}^{n}$ in the solution algebraic equation. In the flow equation, terms $G, A$, and $B$ are unknowns and have explicit values at the $(n-1)$ th time level or iteration for linearization; in the transport equation, $G$, $A$, and $B$ can be calculated according to flow solution and have explicit values at the $n$th time level.

The solid lines in Figs. 2(a) and 3(a) are the nine-node and five-node elements, respectively, used in the computational domain. The finite analytic numerical solutions are derived on the basis of the analytical solutions of the linearized equation in the local element. To obtain the analytical solution for both nine-node and five-node elements, (10) is transformed using $z$ $=z^{*} / \sqrt{G}$ into

$$
\frac{\partial^{2} \Phi^{n}}{\partial x^{2}}+\frac{\partial^{2} \Phi^{n}}{\partial z^{*}}=2 A \frac{\partial \Phi^{n}}{\partial x}+2 B \sqrt{G} \frac{\partial \Phi^{n}}{\partial z^{*}}+g
$$

The elements (solid lines) in the transformed $x$ - $z^{*}$ coordinate system are shown in Figs. 2(b) and 3(b) (with $x$-direction spacings $h_{E}$ and $h_{W}$ and $z^{*}$-direction spacings $h_{S}^{*}$ and $h_{N}^{*}$ ) for the nine-node and five-node elements, respectively. We first create a uniform-spacing element within the transformed element. We choose the smaller spacing in both the $x$ - and $z^{*}$-directions to

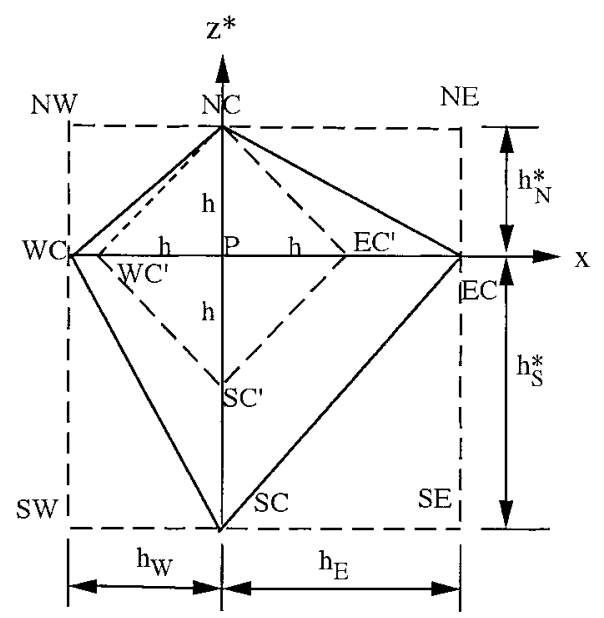

(b)

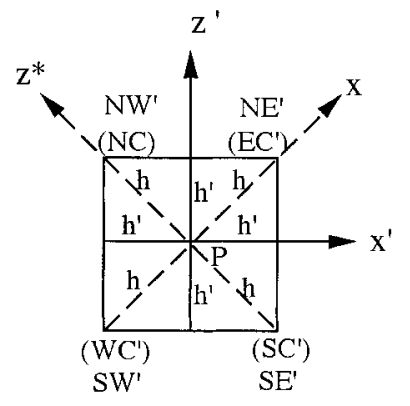

(d)

FIG. 3. Numerical Solution Procedures for Five-Node Element: (a) in Original $x-z$ Coordinate System; (b) Transformation from $z$ Coordinate System to $z^{*}$ Coordinate System; (c) Uniform-Grid Square Element with $45^{\circ}$ Inclination; (d) Uniform-Grid Square Element Used to Provide Analytical Solution 
TABLE 1. Expressions of Locally Linearized Coefficients in Eq. (10)

\begin{tabular}{|c|c|c|}
\hline $\begin{array}{l}\text { Coefficients in Eq. (10) } \\
\text { (1) }\end{array}$ & $\begin{array}{l}\text { Subsurface flow equation } \\
\text { (2) }\end{array}$ & $\begin{array}{l}\text { Subsurface transport equation } \\
\text { (3) }\end{array}$ \\
\hline Dependent variable $\Phi$ & $H$ & $C^{*}=C \theta$ \\
\hline$G$ & 1 & $\frac{\left(D_{z z}\right)_{p}^{n}}{\left(D_{x x}\right)_{p}^{n}}$ \\
\hline$A$ & $-\frac{1}{2 K_{p}^{n-1}}\left[\frac{\partial K(H)}{\partial x}\right]_{p}^{n-1}$ & $\frac{\left(V_{x}-\frac{\partial D_{x x}}{\partial x}-\frac{\partial D_{z x}}{\partial z}\right)_{p}^{n}}{2\left(D_{x x}\right)_{p}^{n}}$ \\
\hline$B$ & $-\frac{1}{2 K_{p}^{n-1}}\left[\frac{\partial K(H)}{\partial z}\right]_{p}^{n-1}$ & $\frac{\left(V_{z}-\frac{\partial D_{z z}}{\partial x}-\frac{\partial D_{z x}}{\partial z}\right)_{p}^{n}}{2\left(D_{z z}\right)_{p}^{n}}$ \\
\hline$g=\operatorname{Re} \frac{\Phi_{p}^{n}-\Phi_{p}^{n-1}}{\left(1-\omega_{p}\right) \Delta t}+f_{p}$ & $\begin{aligned} \operatorname{Re} & =\frac{C_{S}\left(H_{p}^{n-1}\right)}{K_{p}^{n-1}} \\
f_{p} & =-\frac{1}{K_{p}^{n-1}}\left[\frac{\partial K(H)}{\partial z}\right]_{p}^{n-1} \\
& -\frac{C_{S}\left(H_{p}^{n-1}\right)}{K_{p}^{n-1}} \frac{\omega_{p}}{1-\omega_{p}}\left(\frac{\partial H}{\partial t}\right)_{p}^{n-1}\end{aligned}$ & $\begin{aligned} R e & =\frac{1}{\left(D_{x x}\right)_{p}^{n}} \\
f_{p} & =\frac{1}{\left(D_{x x}\right)_{p}^{n}}\left[\left(\lambda R+\frac{\partial V_{x}}{\partial x}+\frac{\partial \mathrm{V}_{z}}{\partial z}\right)_{p}^{n}\left(C^{*}\right)_{p}^{n}\right. \\
& +2\left(D_{x x}\right)_{p}^{n}\left(\frac{\partial^{2} C^{*}}{\partial x \partial z}\right)_{p}^{n-1}-\frac{\omega_{p}}{1-\omega_{p}}\left(\frac{\partial R C^{*}}{\partial t}\right)\end{aligned}$ \\
\hline
\end{tabular}

form the nine-node uniform-spacing element, a rectangular element with spacings $k=h_{N}^{*}$ and $h=h_{E}$, as shown in Fig. 2(c); we also choose the smallest diagonal spacing out of $h_{E}, h_{W}$, $h_{S}^{*}$, and $h_{N}^{*}$ to form the five-node uniform-spacing element, a square element with diagonal spacing $h=h_{N}^{*}, 45^{\circ}$ angle inclined with the $x$-axis, as shown in Fig. 3(c). We further rotate the $x-z^{*}$ coordinate system a $45^{\circ}$ angle counterclockwise; then, the five-node element in the new $x^{\prime}-z^{\prime}$ coordinate system can be viewed as given in Fig. 3(d). The analytical solution of (12) in the uniform-grid elements, as in Figs. 2(c) and 3(d), is readily available, provided that appropriate boundary functions are given. According to Chen and Chen (1984a,b), the linear and exponential function is an appropriate boundary function for each side (three nodes) of the nine-node element, and the exponential function is proper for each side (two nodes) of the five-node element. Following the above solution procedures, detailed by Carlson (1993) and Tien (1992), the finite analytic numerical solutions of (12) in the fictitious uniform-grid elements for both nine-node and five-node elements can be obtained. The obtained solutions can be further used to extrapolate the nodal values of the original nonuniform-grid element based on the proposed boundary functions. The algebraic equation that links nodal variables in the local element can thus be established. When the obtained analytical solution in each element is imposed on the interior node $P$, it yields

$$
\Phi_{p}^{n}=\sum_{i=1}^{8} c_{i} \Phi_{i}^{n}+c_{p} \Phi_{p}^{n-1}+\Phi_{s o}^{9}
$$

for the nine-node element, and

$$
\Phi_{p}^{n}=\sum_{j=1}^{4} b_{j} \Phi_{j}^{n}+b_{p} \Phi_{p}^{n-1}+\Phi_{s o}^{5}
$$

for the five-node element. Here, index $i$ denotes the eight boundary nodes of the nine-node element, four at the corners and four at the middle of each side; and index $j$ denotes the four corner nodes of the five-node element; while $\Phi_{s o}^{5}$ and $\Gamma_{s o}^{2}$ are the source terms. The expressions of the finite analytic coefficients $c_{i}, c_{p}, b_{j}, b_{p}$ and the source terms $\Phi_{s o}^{9}$ and $\Phi_{s o}^{5}$ are detailed by Carlson (1993) and Tien (1992). Eqs. (13) and (14) are linear algebraic equations that relate the nodal variables in the local element. Eqs. (13) and (14) can be derived for each computational node to form a system of algebraic equations.

\section{Numerical Features}

It should be remarked here that, in the execution of numerical computations, the five-node elements used for irregular boundaries can be covered by the nine-node elements. Eventually, there are four fictitious corner nodes with zero magnitude of finite analytic coefficients in the five-node element. With this arrangement, the linear algebraic equations for the five-node element and the nine-node element can be expressed by the same form, (13). The linearized coefficients $A, B$, and $G$ and the element spacings are major factors affecting the finite analytic coefficients. The terms $A h_{w}, A h_{E}, B \sqrt{G} h_{N}$, and $B \sqrt{G} h_{S}$ are used to calculate the finite analytic coefficients (Tien 1992; Carlson 1993). These terms are physically equivalent to the Peclet number, which is defined as (Advection)(Grid Spacing)/Diffusion. The Peclet number is usually used as an index for evaluation of numerical accuracy in the advection-diffusion partial differential equations and groundwater flow problems (Hwang et al. 1985; Leonard and Mokhtari 1990). It should be noted that $G$ is equal to one in the unsaturated flow equation, while $G$ is not one in the solute transport equation.

\section{MODEL VERIFICATIONS}

\section{Unsaturated Flow}

The modeling unsaturated flow using the finite analytic method was first reported by Tsai et al. (1993). In the study, the obtained finite analytic numerical solutions were verified with the finite element solutions of FEMWATER code (Yeh and Ward 1980; Yeh 1987). The currently developed numerical model is aimed at improving the capability of capturing the sharp wetting front of unsaturated flow. The large velocity gradient associated with the sharp wetting front in an unsaturated zone is very significant and may deteriorate the accuracy of numerical solutions. Therefore, the spatial weighting schemes are proposed to evaluate the averaged unsaturated hydraulic conductivity in a discretized element to improve the numerical accuracy.

The constitutive function describing the relationship of the unsaturated hydraulic conductivity and pressure head for soil is highly nonlinear. Besides, the linearized coefficients $G, A$, $B$, and $g$, as shown in (10), are functions of unsaturated hydraulic conductivity and porous velocity (see Table 1 ). Therefore, these linearized coefficients are intimately related to the 
constitutive function. Consequently, the spatial weighting scheme is significant to numerical performance in capturing sharp wetting front. In the following, nine schemes are proposed. These schemes are similar to those proposed by Haverkamp and Vauclin (1979), who used the weighting interblock hydraulic conductivity values to model one-dimensional unsaturated flow. Among the nine schemes, type 1 is the central node expression that is commonly used in the finite analytic method (Tsai et al. 1993) and also the expression of the linearized coefficients in Table 1; type 2 is the arithmetic mean expression and type 3 adds an extra central weighting factor to type 2 ; type 4 is the geometric mean expression and type 5 adds an extra central weighting factor to type 4; type 6 is the harmonic mean expression; and types 7-9 are the upstream weighting schemes associated with arithmetic mean, geometric mean, and harmonic mean, respectively. The large gradients mainly arise along the vertical direction caused by the gravitational effect. Here a vertical, one-dimensional infiltration test is used for the model verification. All the nine schemes, as listed in Table 2, are examined by comparing associated numerical experiments with infiltration data provided by Touma and Vauclin (1986). It should be noted that in Table 2 the subscript $i$ of the hydraulic conductivity $K$ denotes the central node of the three vertically consecutive nodes, or an element, and $i-1$ and $i+1$ refer to the upper and lower nodes of the element, respectively.

Times increment $\Delta t=10^{-4} \mathrm{~h}$ is used for computation, and the total simulated time is $0.4 \mathrm{~h}$. The simulated results are compared with laboratory data observed by Touma and Vauclin (1986), and moisture contents in the vertical at time 0.4 $\mathrm{h}$ are shown in Fig. 4. It should be noted that the moisture front from beginning to time $0.4 \mathrm{~h}$ is very sharp because the initial moisture of the sandy soil was very dry. The computational grid size, $\Delta z$, is $1.0 \mathrm{~cm}$. Fig. 4(a) shows that the numerical solutions obtained based on the types 4, 5, and 9 weighting schemes agree with the laboratory data better than the others, but none of them perfectly fits the laboratory data. For $\Delta z=0.5 \mathrm{~cm}$, numerical solutions obtained based on types 4 and 5 weighting schemes fit the laboratory data perfectly, as shown in Fig. 4(b). This is still true even when the computational grid size is reduced to $0.2 \mathrm{~cm}$, as shown in Fig. 4(c). Nevertheless, all nine numerical solutions are stable, and no significant difference of computing time among them is found.

The above findings suggest the geometric mean spatial weighting scheme be used for evaluation of averaged unsatu-

TABLE 2. Nine Weighting Schemes Used to Evaluate Averaged Unsaturated Hydraulic Conductivity in Discretized Element

\begin{tabular}{c|l|l}
\hline \hline $\begin{array}{c}\text { Type } \\
(1)\end{array}$ & \multicolumn{1}{|c|}{$\begin{array}{c}\text { Weighting scheme } \\
(2)\end{array}$} & \multicolumn{1}{c}{$\begin{array}{c}\text { Mathematical expression } \\
(3)\end{array}$} \\
\hline 1 & Central node & $K=(K)_{i}$ \\
\hline 2 & Arithmetic mean & $K=\left[(K)_{i-1}+(K)_{i}+(K)_{i+1}\right] / 3$ \\
\hline 3 & $\begin{array}{c}\text { Arithmetic mean with extra } \\
\text { central weighting }\end{array}$ & $K=\left[(K)_{i-1}+2(K)_{i}+(K)_{i+1}\right] / 4$ \\
\hline 4 & Geometric mean & $K=\sqrt[3]{(K)_{i-1}(K)_{i}(K)_{i+1}}$ \\
\hline 5 & $\begin{array}{l}\text { Geometric mean with extra } \\
\text { central weighting }\end{array}$ & $K=\sqrt[4]{(K)_{i-1}(K)_{i}^{2}(K)_{i+1}}$ \\
\hline 6 & $\begin{array}{l}\text { Harmonic mean } \\
\text { armenthe }\end{array}$ & $K=3 /\left[\frac{1}{(K)_{i-1}}+\frac{1}{(K)_{i}}+\frac{1}{(K)_{i+1}}\right]$ \\
\hline 8 & $\begin{array}{l}\text { Upstream weighting with } \\
\text { geometric mean }\end{array}$ & $K=\sqrt{(K)_{i-1}(K)_{i}}$ \\
\hline 9 & $\begin{array}{c}\text { Upstream weighting with } \\
\text { harmonic mean }\end{array}$ & $K=2 /\left[\frac{1}{(K)_{i-1}}+\frac{1}{(K)_{i}}\right]$ \\
\hline \hline
\end{tabular}

rated hydraulic conductivity in an unsaturated flow problem. Since both flow and solute transport are the advection-diffusion equations, the finite analytic numerical formulations are the same. Therefore, the geometric mean weighting scheme will be used for both flow and transport equations in the subsequent numerical verifications and applications.

\section{Subsurface Transport with Spatially Variable Coefficients}

Due to the lack of an analytical solution for subsurface solute transport in an unsaturated zone, an equivalent transport problem in a saturated zone with spatially variable advection and dispersion coefficients is adopted for verification. The selected example is a slug of pollutant with a unit mass released at $x=x_{o}$ and $z=z_{o}$ in a nonuniform flow field. The dispersion coefficients are assumed as a quadratic function of distance such that $D_{x x}=D_{o} x^{2}$ and $D_{z z}=D_{o} z^{2}$. The field velocities are assumed of a linear function of distance $V_{x}=-V_{o} x, V_{z}=-V_{o} z$. The analytical solution (Carslaw and Jaeger 1971) is available for (4) under the special condition of $\theta=1, R=1, D_{x z}=D_{z x}$ $=0, \lambda=0$, and $s=0$ as

$$
\begin{aligned}
& C\left(x, z, t, x_{o}, z_{o}\right)=\frac{1}{4 \pi t D_{o}} \\
& \cdot \exp \left\{\frac{-\left[\ln \left(x / x_{o}\right)+\left(V_{o}+D_{o}\right) t\right]^{2}-\left[\ln \left(z / z_{o}\right)+\left(V_{o}+D_{o}\right) t\right]^{2}}{4 t D_{o}}\right\}
\end{aligned}
$$

Eq. (15) describes the evolution of solute concentration $C$ with time $t$ for the slug of pollutant in the nonuniform velocity field. The parameters used for analytical solution in (15) are $V_{o}=$ $1.0 /$ year, $D_{o}=1.0 /$ year, $x_{o}=4.0 \mathrm{~km}, z_{o}=4.0 \mathrm{~km}$, and $t=0.2$ year. The temporal and spatial increments used for computation are $\Delta t=0.002$ year, $\Delta x=0.02 \mathrm{~km}$, and $\Delta z=0.02 \mathrm{~km}$ for both nine-node and five-node elements. This condition refers to a transient transport problem with a maximal Peclet number of one. It is useful to investigate numerical accuracy when the five-node elements are involved. The nine-node elements are placed in the interior domain, and $N$-row and $N$-column fivenode elements are positioned at boundaries, as shown in Fig. 5. The element layout is essentially designed for assessing accuracy of the five-node elements in a domain with irregular boundaries. There are four different $N$ values used in the numerical experiments, i.e., $N=0,2,5$, and 10. The simulated results are shown in Fig. 6. For $N=0$, only the nine-node elements are used for numerical simulation. The simulated contours of the solute concentration are plotted in solid line, as presented in Fig. 6(a). The contours agree very well with those of the analytical solution plotted in a dashed line. Fig. 6(b) refers to $N=2$, and no visible difference is found as compared with the numerical solution of $N=0$. The numerical solution of $N=5$ is presented in Fig. 6(c), the contours of which are slightly different from the numerical solutions of $N$ $=0$ and 2 . When $N$ is increased to 10 , the simulated contours of solute concentration, as shown in Fig. 6(d), deviate significantly from those of the analytic solution. The average nodal errors described by the solute concentration compared with the analytical solutions are $0.0022,0.0025,0.0031$, and 0.015 for $N=0,2,5$, and 10 , respectively. The finite analytic method used here is not an integral method; therefore, the global mass conservation is not guaranteed. Adequate conservation of global mass is a necessary but not a sufficient condition for acceptability of a numerical simulator. Nevertheless, the ratios of global mass conservation associated with Fig. 6 are found in between 99.0 and $99.9 \%$. This indicates that the numerical accuracy is directly related to nodal error but may not be closely related to the global mass conservation in the finite analytic method. 
Moisture Content

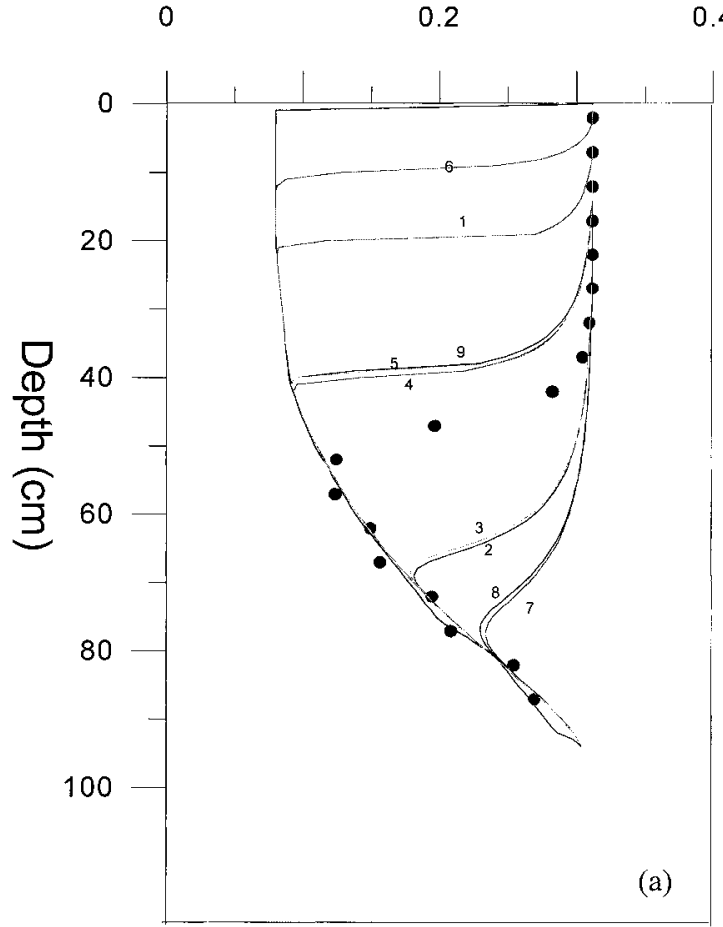

Moisture Content

4

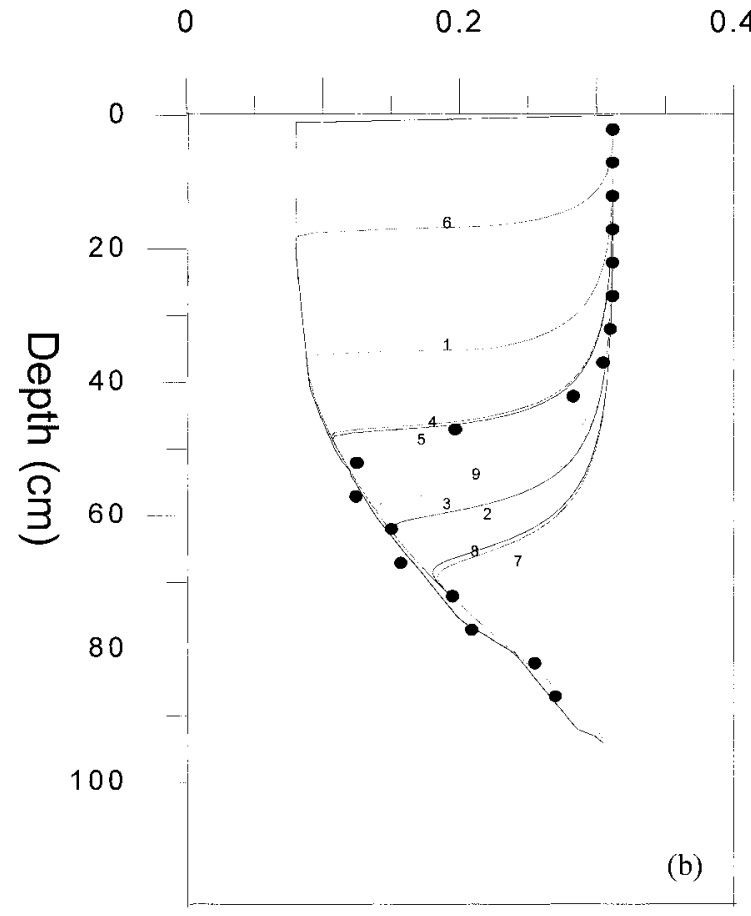

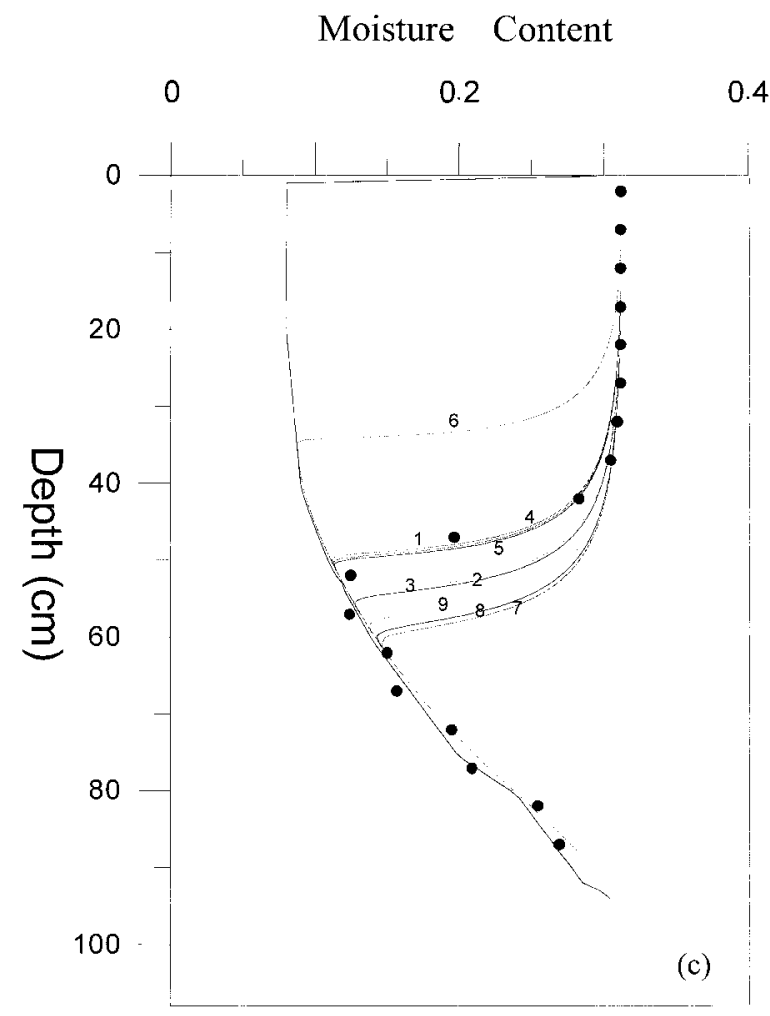

FIG. 4. Numerical Experiments on Nine Weighting Schemes Used to Evaluate Unsaturated Hydraulic Conductivity: (a) $\Delta z=1.0 \mathrm{~cm}$; (b) $\Delta z=0.5 \mathrm{~cm}$; (c) $\Delta z=0.2 \mathrm{~cm}$ (•: Experimental Data; 1: Central Node; 2: Arithmetic Mean; 3: Arithmetic Mean with Extra Central Weighting; 4: Geometric Mean; 5: Geometric Mean with Extra Central Weighting; 6: Harmonic Mean; 7: Upstream Weighting with Arithmetic Mean; 8: Upstream Weighting with Geometric Mean; 9: Upstream Weighting with Harmonic Mean)

Based on the above findings, we can see that the advantage of using the five-node element is that we may match the irregular boundaries while remaining on the Cartesian coordinate system. Therefore, the system of algebraic equations can be solved through simple, straightforward, line-by-line iteration. The disadvantage is that the accuracy of the five-node element is not as good as that of nine-node element, especially in the case that the flow advection comes from a direction with an inclined $45^{\circ}$ angle to the $x$ or $z$ coordinates. However, for the purpose of matching the irregular boundaries, only very few five-node elements placed at the boundaries are required. In general, only one five-node element is needed. Eventually, it would not deteriorate the overall accuracy. The comparisons noted above indicate that the finite analytic method is capable 


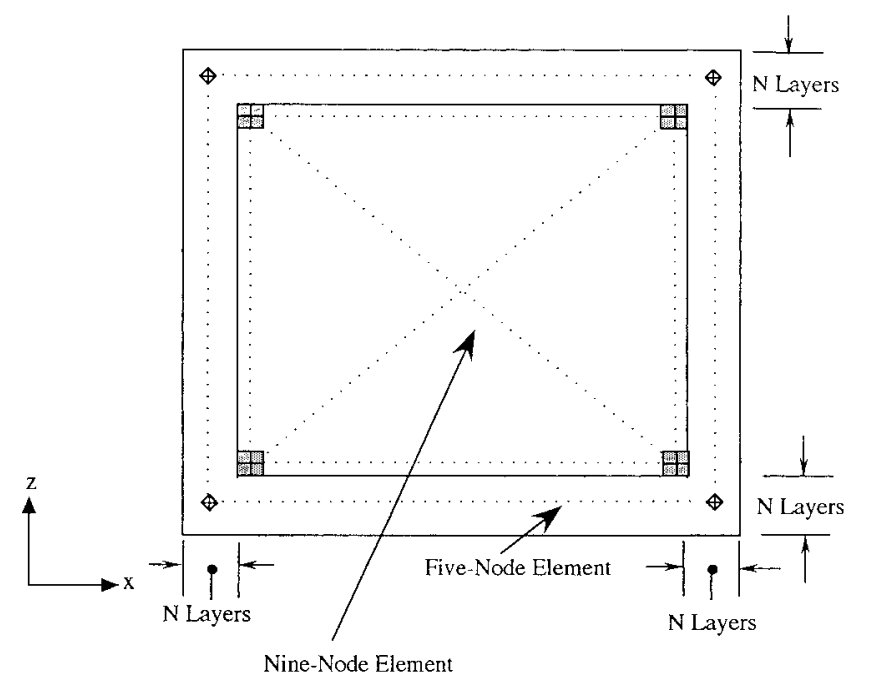

FIG. 5. Layout of Computational Grids Using Nine-Node Elements Placed in Interior Domain and Using $N$ Layers of FiveNode Elements Placed at Boundaries for Verification of Solute Transport Problem
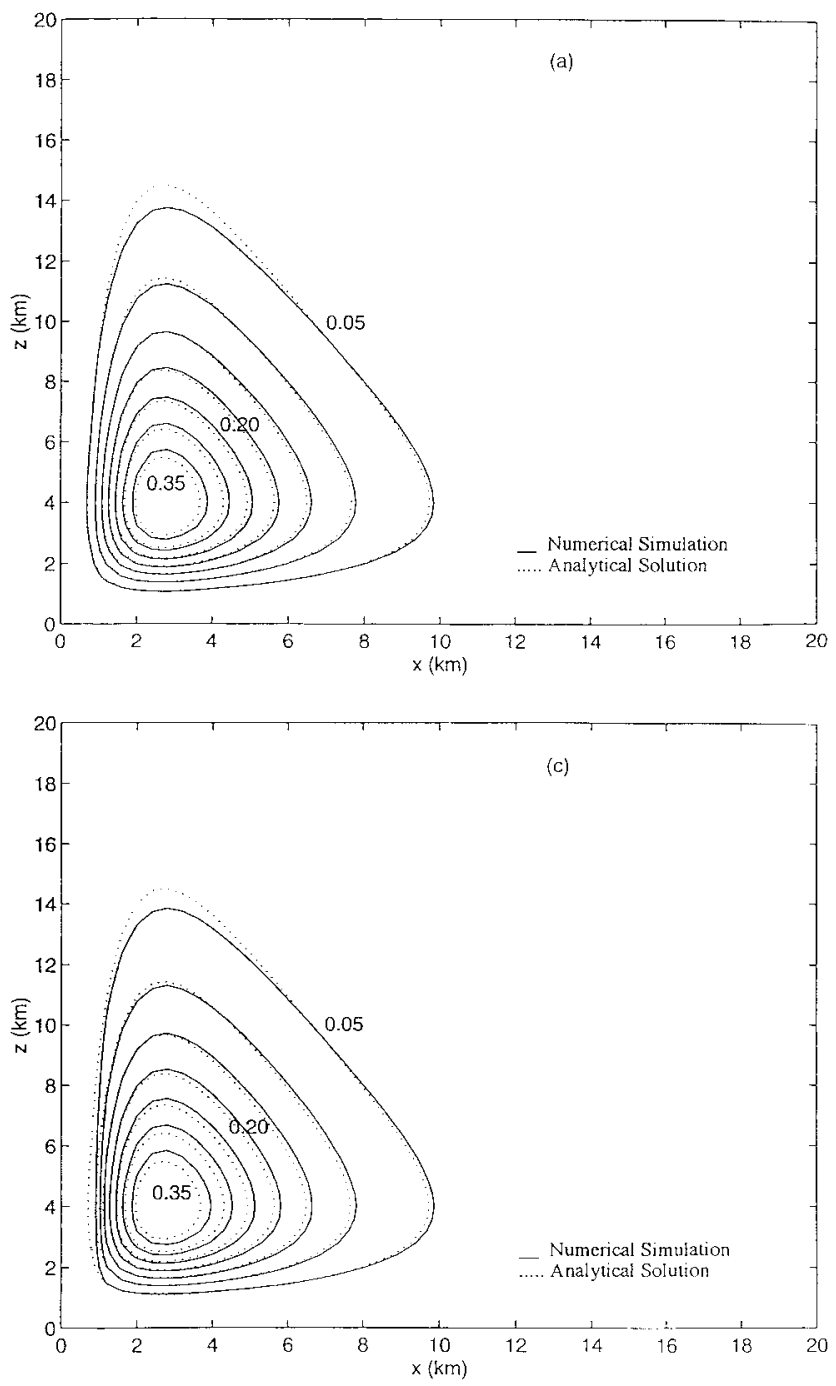

of simulating solute transport problems with spatially variable advection and dispersion coefficients under a presumed condition of geometric irregularity by using mixed nine-node and five-node elements. In an unsaturated zone, the nonlinear behavior of fluid flow and solute transport acts in a similar way to the nonuniform advective and dispersive processes in a saturated zone. Therefore, the verification addressed above is transposable for solute transport in an unsaturated zone.

\section{NUMERICAL ASSESSMENT OF SOLUTE MIGRATION FROM LANDFILL IN UNSATURATED ZONE}

\section{Problem Description}

In the following example, the solute migration along the vertical in unsaturated zone is presented. The ground-water table is situated below the landfill, as sketched in Fig. 7. To protect the natural environment of ground water, it is important to estimate the travel time of the polluted solute from reaching the ground-water table and predict the potential spatial distribution of solute concentration in the unsaturated zone. The illustrated example entails a landfill with an irregular ground surface. During the rainfall season, the solute water within the landfill may infiltrate into the unsaturated zone and propagate downward. Finally, the polluted solute may reach the groundwater table and contaminate ground water.
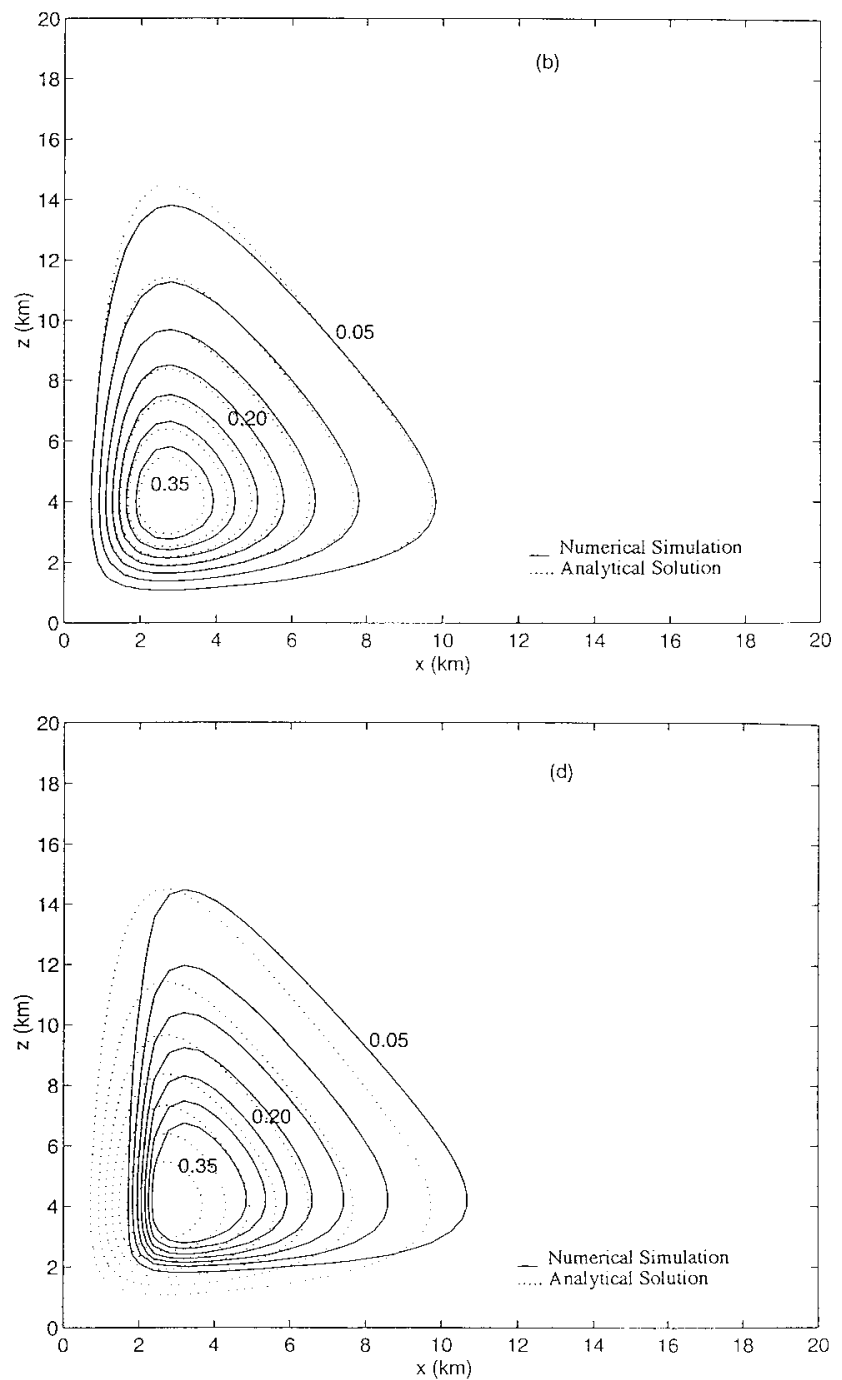

FIG. 6. Comparison of Contours of Solute Concentration at Time $=0.4$ Years in Verification Problem between Finite Analytic Numerical Solution and Analytical Solution Using $N$ Layers of Five-Node Elements Placed at Boundaries of Computational Domain: (a) $N=0$; (b) $N=2$; (c) $N=5$; (d) $N=10$ 

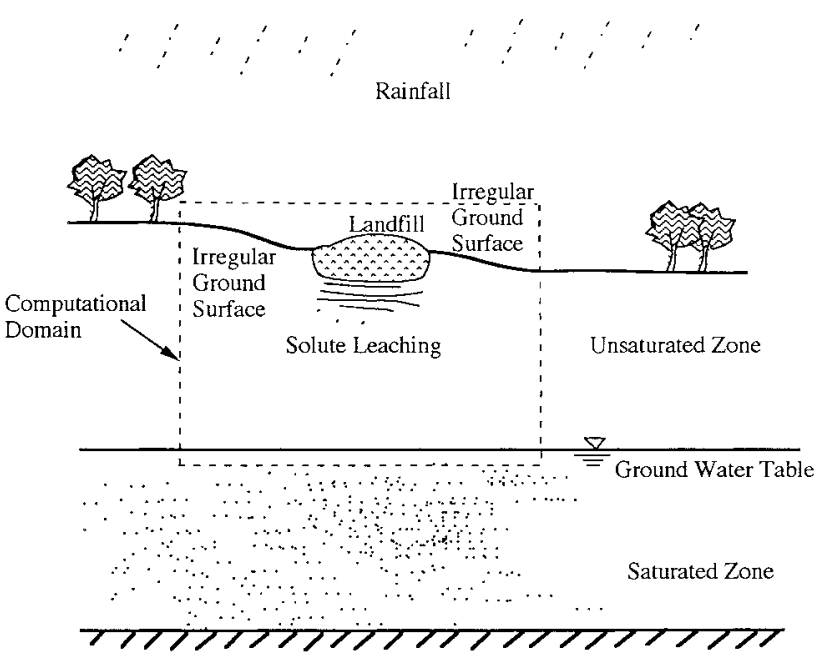

FIG. 7. Schematic Representation of Solute Plume Released from Landfill with Irregular Ground Surface

In the present study, the exponential forms of the constitutive functions of soil, $K(H)$ and $\theta(H)$, are used (Warrick and Lomen 1976). $K(H)$ and $\theta(H)$ are given, respectively, as

$$
K(H)=K_{o} \exp (\beta H)
$$

and

$$
\theta(H)=\left(K_{o} / A_{o}\right) \exp (\beta H)
$$

in which $K_{o}$ and $A_{o}$ are empirical constants for the soil. $K_{o}=$ $0.0020 \mathrm{~cm} / \mathrm{min}, \beta=0.01$, and $A_{o}=0.004 \mathrm{~cm} / \mathrm{min}$. These parameters refer to a low permeability of soil like clay or silt, which can be placed beneath the landfill to reduce the infiltration rate. The bulk density of the clay loam, $\rho_{b}$, is $1.55 \mathrm{~g} / \mathrm{cm}^{3}$. The dispersivity of soil for solute transport is scale dependent. Transversal dispersivity $\alpha_{t}$ ranges typically from 10 to $40 \%$ of longitudinal dispersivity $\alpha_{1}$. For the scale shown in Fig. 7, $\alpha_{1}$ $=10 \mathrm{~m}$ and $\alpha_{t}=3 \mathrm{~m}$ are reasonable assumptions for the saturated zone (Anderson 1979). Here, we assume it is also applicable for the unsaturated zone.

To predict the fluid flow and solute transport in the unsaturated zone for a domain with irregular boundaries, as enclosed by the dashed line in Fig. 7, the five-node and ninenode elements are used to form the grid system in the computational domain. During the rainfall season, the water flux leaking from the bottom of the landfill into the unsaturated zone is taken as $0.024 \mathrm{~cm} / \mathrm{hr}$. This amount of flux is small due to the low permeable substance placed on the bottom of the landfill. In addition, the water flux from the sloping ground surface infiltrating into the unsaturated zone is $0.12 \mathrm{~cm} / \mathrm{hr}$. The boundary conditions and computational grids used for simulation can be found in Tsai et al. (1993).

The initial condition of the pressure head, $H$, in the unsaturated zone is assumed $-100 \mathrm{~cm}$ beneath the ground surface and is linearly distributed between the ground surface and the ground-water table. The initial solute concentration is zero everywhere. Variable time increments are used in the computation. Three-hour time intervals are used for the first day, and 24-hour time intervals are used thereafter. For each time level, the solution is obtained by the line-by-line iteration in the $z$ direction from the low to the top boundaries until the difference of $H$ and $C^{*}(=C \theta)$ between successive iterations is less than $0.001 \mathrm{~cm}$ and 0.00001 , respectively. It is a two-step numerical procedure. The unsaturated flow equation, (1), is solved first. Then the output, including the flow field and moisture contents obtained by solving (1), is taken as an input for solving the solute transport equation, (2).

\section{Results and Discussion}

The contours of solute concentration in the unsaturated zone for times equal to 15 and 30 days are shown in Figs. 8(a and b), respectively. These two figures reveal that transversal migration of solute is not so significant as compared with the longitudinal (downward) migration. Besides, the solute concentration 0.1 is found to reach the ground-water table at 30 days of travel time. Another finding of the numerical solution of unsaturated flow is that the finite analytic coefficients within a local element are all positive and the summation is one. The finite analytic coefficients for the numerical solutions of solute transport are all positive as well. Although the physical decay coefficient, $\lambda$, is zero here, the numerical decay coefficient $\left[\lambda+(1 / R)\left(\partial V_{x} / \partial x\right)+(1 / R)\left(\partial V_{z} / \partial z\right)\right]$, s seen in Table 1 , is a nonzero term. Therefore, the summation of the finite analytic coefficients in an element is found to be less than, but close to, one. The maximal Peclet numbers in the simulation of unsaturated flow and solute transport are found to be about 0.6 and 1.2, respectively, Magnitude of the Peclet number near this range is close to the standard suggested by Leonard and Mokhtari (1990) for consideration of satisfactory numerical accuracy.

From the simulation and results of the landfill applications, we found some advantages of the numerical model. Since the finite analytic method is an analytically based method, the truncation error can be minimized, and the numerical stability
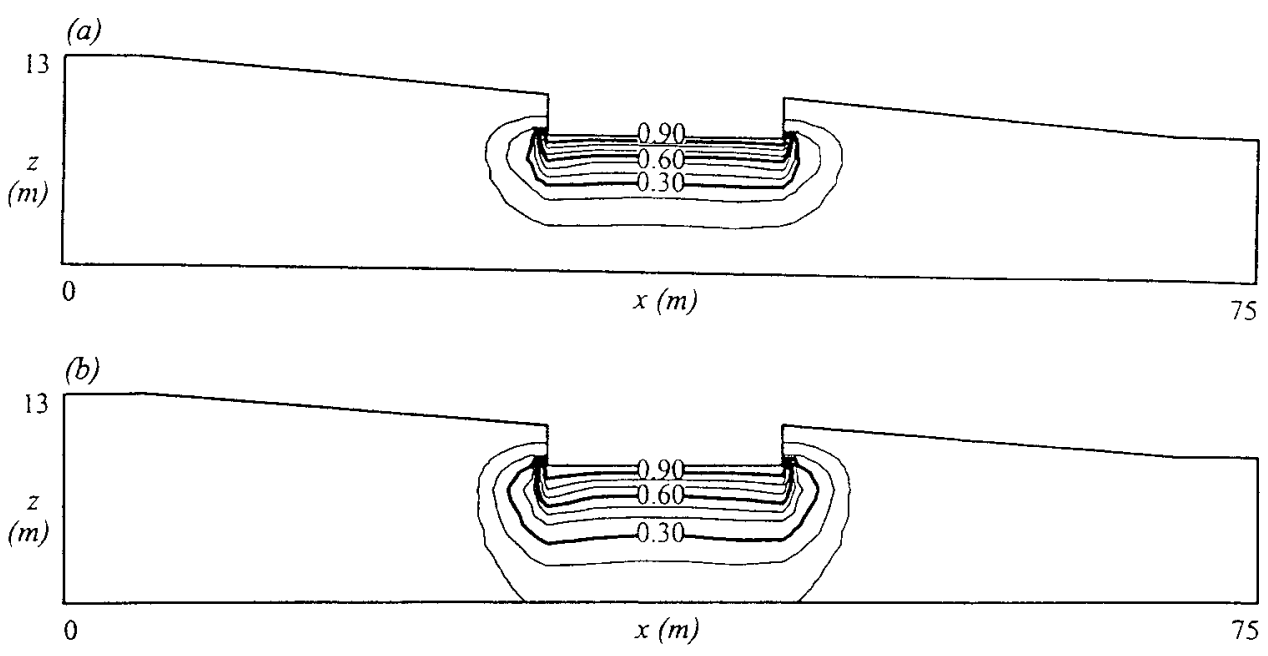

FIG. 8. Contours of Solute Concentration Obtained from Simulation of Landfill Leaching Using $K_{d}=0$ and $\lambda-0$ : (a) Time $=15$ Days; (b) Time $=30$ Days 
is robust. Because the advection-diffusion equation is the basic form for deriving the finite analytic numerical solution and the flow and transport equations in the unsaturated zone are both the advection-diffusion partial differential equations, only one formulation is required but applied to both. This feature enhances the numerical efficiency. Moreover, the current numerical model is shown to be able to simulate practical problems like solute transport under a landfill in an unsaturated zone with an irregular ground surface by proper use of mixed fivenode and nine-node elements. Since the structured-grid system is used, the layout of the grid is straightforward and the simple line-by-line iteration can be used for convergence process.

Some minor numerical disadvantages may still exist in the above applications. First, the accuracy of the five-node element and the upwinding effect among nodes are worse than those of the nine-node element. Fortunately, only one fivenode element is required to place at the irregular boundaries. Secondly, the use of five-node and nine-node elements in a structured-grid system may increase the total number of computational nodes and, hence, the computing time. Finally, the adaptive grid associated with the refinement of the local grid may be not easily accessible to the proposed structure-grid system, and further development is required.

\section{CONCLUDING REMARKS AND SUGGESTIONS}

The purpose of the present study is to invoke the finite analytic method to solve the vertical, two-dimensional subsurface flow and transport equations in the unsaturated zone. In the finite analytic method, the coefficients of the governing equations are frozen in each element, so that linear partial differential equations can be obtained and solved. A spatial weighting scheme is used to evaluate the averaged unsaturated hydraulic conductivity in the discretized element. The geometric mean schemes, among the nine potential spatial weighting schemes, are found to better capture the sharp wetting front of the infiltration data. In solving the subsurface transport problem, this study illustrates how to use mixed five-node and nine-node elements to match a domain with irregular boundaries and achieve the required numerical accuracy. The applications of the developed numerical model to the landfill demonstrate that the finite analytic numerical solutions for subsurface flow and transport in the unsaturated zone preserve the nature of mass decay, result in a matrix of the positive type, increase the mesh Peclet number of stability, and feature an unconditionally stable scheme. Moreover, the use of the structured-grid system enables the line-by-line iteration to be readily applicable in solving the matrix of the system of the algebraic equation. However, the disadvantage associated with an adaptive grid may arise when dealing with the local refinement of the grids. Furthermore, to achieve the optimal numerical performance, an effective grid generation algorithm enabling proper use of the five-node and nine-node elements to automatically match irregular boundaries of the computational domain would be very useful.

\section{ACKNOWLEDGMENTS}

The study was funded by the National Science Council, Taiwan under Grant No. NSC-86-2211-E-321-001.

\section{APPENDIX I. REFERENCES}

Anderson, M. P. (1979). "Using models to simulate the movement of contaminants through groundwater systems." Critical Rev. in Envir. Control, 9(2), 97-156.

Bear, J. (1979). Hydraulics of groundwater water. McGraw-Hill, New York, 213-234.

Carslaw, H. S., and Jaeger, J. C. (1971). Conduction of heat in solids. Oxford University Press, London.

Carlson, K. D. (1993). "Finite analytic numerical simulation of two- dimensional flow problems with irregular boundaries in Cartesian coordinates," MS thesis, Dept. of Mech. Engrg., University of Iowa, Iowa City, Iowa.

Chen, C. J. (1989). "Finite analytic numerical method." Handbook of numerical heat transfer, Wiley, New York.

Chen, C. J., and Chen, H. C. (1984a). "Development of finite analytic numerical method for unsteady two-dimensional Navier-Stokes equation." J. Comp. Physics, 53(2), 209-226.

Chen, H. C., and Chen, C. J. (1984b). "Development of finite analytic numerical method for unsteady three-dimensional Navier-Stokes equations: computation of internal flows." ASME FED, 14, 159-165.

Haverkamp, R., and Vauclin, M. (1979). "A note on estimating finite difference interblock hydraulic conductivity values for transient unsaturated flow problems." Water Resour. Res., 15(1), 1354-1360.

Hwang, J. C., Chen, C. J., Sheikhoslami, M., and Panigrahi, B. K. (1985). "Finite analytic numerical solution for two-dimensional groundwater solute transport." Water Resour. Res., 21(90), 1354-1360.

Leonard, B. P., and Mokhtari, S. (1990). "Beyond first-order upwinding: the ultra-sharp alternative for non-oscillatory steady-state simulation of convection." Int. J. Numer. Methods in Engrg., 30, 729-766.

Li, W., Xie, Q., and Chen, C. J. (1989). "Finite analytic solution of flow over spillways." J. Engrg. Mech., ASCE, 115(12), 2635-2647.

$\mathrm{Pi}, \mathrm{Z}$., and Hjelmfelt Jr., A. T. (1994). "Hybrid finite analytic solution of lateral subsurface flow." Water Resour. Res., 30(5), 1471-1478.

Tien, H. C. (1992). "Finite analytic method for computational domain with irregular boundaries," PhD thesis, Dept. of Appl. Math., University of Iowa, Iowa City, Iowa.

Touma, J., and Vauclin, M. (1986). "Experimental and numerical analysis of two phase infiltration in a partially saturated soil." Transport in Porous Media, 1, 28-55.

Tsai, W. F., Chen, C. J., and Tien, H. C. (1993). "Finite analytic numerical solutions for unsaturated flow with irregular boundaries." J. Hydr. Engrg., ASCE, 119(11), 1274-1298.

Warrick, A. W., and Lomen, D. O. (1976). "Time-dependent linearized infiltration. III: Strip and disc sources." Soil Sci. Soc. Am. J., 40, 639643.

Yeh, G. T. (1987). "FEMWATER: a finite-element model of water flow through saturated-unsaturated porous media." Rep. ORNL-5567/R1, Oak Ridge National Laboratory, Oak Ridge, Tenn.

Yeh, G. T., and Ward, D. S. (1980). "FEMWATER: a finite-element model of water flow through saturated-unsaturated porous media." Rep. ORNL-5567, Oak Ridge National Laboratory, Oak Ridge, Tenn.

Zeng, X. J., and Li, W. (1987). "The stability and convergence of finite analytic method for unsteady two-dimensional convective transport equations." Turbulence measurements and flow modeling, C. J. Chen, L. D. Chen, and F. M. Holly, eds., Hemisphere Publishing, Bristol, Pa., 427-433.

\section{APPENDIX II. NOTATION}

The following symbols are used in this paper:

$A=x$-direction locally linearized advective coefficient in basic form of finite analytic equation;

$B=z$-direction locally linearized coefficient in basic form of finite analytic equation;

$b_{i}=$ finite analytic coefficients of five-node solution algebraic equation;

$C=$ solute concentration;

$C_{S}=$ specific moisture capacity;

$C^{*}=C \theta$

$c_{i}=$ finite analytic coefficients of nine-node solution algebraic equation;

$G=z$-direction diffusion parameter shown in basic form of finite analytic equation;

$g=$ source function in locally linearized governing equation;

$H=$ pressure head;

$h=$ horizontal grid spacing used in element;

$K=$ hydraulic conductivity;

$K_{d}=$ distribution coefficient;

$k=$ vertical grid spacing used in element;

$n=n$th time step;

$P=$ central node in rectangular local element;

$R=$ retardation factor; 
$T_{1 / 2}=$ half-life time of mass decay;

$t=$ time;

$x, z=$ spatial coordinates;

$x^{\prime}, z^{\prime}=45^{\circ}$ rotational spatial coordinates;

$z^{*}=$ transformed coordinate of $z$ coordinate;

$\alpha_{l}=$ longitudinal dispersivity;

$\alpha_{t}=$ transversal dispersivity;
$\Delta t=$ time interval;

$\Delta x=$ grid spacings used in $x$ direction;

$\Delta z=$ grid spacings used in $z$ direction;

$\theta=$ volumetric water content;

$\lambda=$ first-order decay coefficient;

$\Phi=$ dependent variable of finite analytic solution; and $\omega=$ time-weighting factor. 